\title{
POS Tagging of Gujarati Text using VITERBI and SVM
}

\author{
Manisha Prajapati \\ PhD Scholar, \\ Gujarat Technological University \\ Ahmedabad, Gujarat, India
}

\author{
Archit Yajnik, PhD \\ Associate Professor \\ SMIT, Sikkim, India
}

\begin{abstract}
Grammatical feature (POS) Labeling is a testing undertaking to distinguish the significance of each word in a sentence. This paper shows the assignment of distinguishing Grammatical form TAG for each transform in a Guajarati sentence utilizing the system of support Vector Machine and Viterbi deciphering method. Guajarati corpus of 1700 words is taken and tried it precisely. Labeling is done utilizing Viterbi and SVM and the outcome is examined in four classifications. In every one of the classifications Viterbi based method gives much better correctness's.
\end{abstract}

\section{Keywords}

SVM, Tagged Corpus, BISTag set, Viterbi.

\section{INTRODUCTION}

POS tagging is the way toward doling out a grammatical feature, similar to thing, verb, pronoun, intensifier, qualifier or other lexical class marker to each word in a sentence. The settling of vagueness in POS labeling framework is testing assignment for all-Normal Dialect Preparing (NLP) scientists. The contribution to a labeling calculation is a series of expressions of a characteristic dialect sentence and a particular label set the yield is a solitary POS Tag for each word. There are distinctive machine learning ways to deal with the issue of allocating each expression of a content with a sections of discourse tag, which is known as POS labeling. In this paper the execution of a POS Tagger for Guajarati dialect is indicated utilizing SVM. Support Vector Machine is fundamentally utilized for grouping and perceives the example. SVMs have high speculation execution autonomous of measurement of highlight vectors. When all is said in done, Labeling is the way toward doling out any name to a phonetic unit or token. The phonetic unit might be word, express, sentence and so on. In this work the labeling alludes to the way toward relegating grammatical form (POS) tag to a word. The PC programs intended to consequently appoint the POS tag to a word in regular dialect content, are called taggersParts of discourse labeling is the way toward increasing the words in a characteristic dialect sentence as comparing to a specific grammatical form labels or lexical classes or word classes, in light of the two its definition, and additionally its unique situation. Support vector machines (SVM) have turned into a mainstream device for discriminative order. For the most part labeling is required to be as exact as could be expected under the circumstances and as proficient as could reasonably be expected. The SVM Device is planned to agree to every one of the necessities of current NLP innovation, by joining straightforwardness, adaptability, power, convenience and productivity with cutting edge precision. This is accomplished by working in the SVM learning outline work and by offering NLP inquires about a profoundly adjustable consecutive tagger generator [1]. We have connected the SVM Tool to the issue of grammatical feature (POS) labeling. POS labeling can be utilized as a part of Content to Discourse (TTS) applications, data recovery, parsing, data extraction, interpretation and some more. This paper begins with the hypothesis of support Vector Machines (SVM) and later clarifies about how SVM Tool can be connected to the issue of pos labeling. Preparing and testing information is gathered from the Guajarati daily paper. The block diagram is depicted in Fig. 1. Block diagram of POS tagging for Gujarati Text

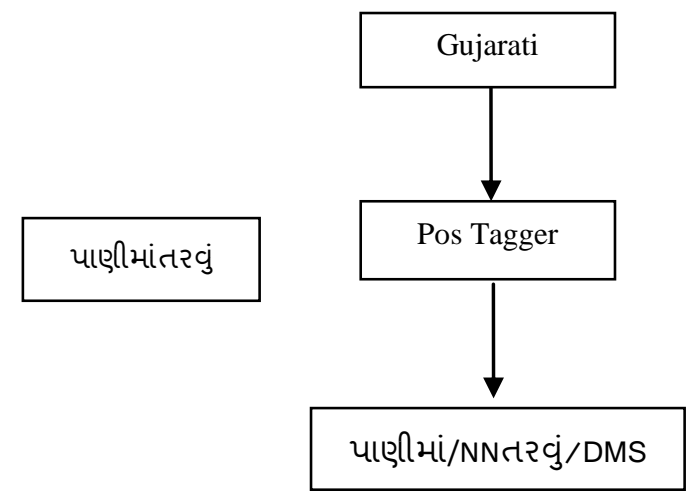

\section{METHODOLOGY}

\subsection{Support Vector Machine}

In their essential frame, SVM build the hyper plane in input space that accurately is olates the case information into two classes. Subsequently SVM is a parallel classifier. This hyper plane can be utilized to make the expectation of class for concealed information. The hyper plane dependably exists for the straightly distinguishable information [18].

\subsection{Viterbi}

Viterbi algorithm try to determine the most likely sequence of states called the Viterbi path that results in a sequence of observed events especially in hidden Markov model. The number of possible paths grows exponentially with the length of the input sequence. viterbi algorithm is dynamic programming algorithm, used to find the optimal state sequence in polynomial time [2].

\section{EXPERIMENTAL DETAILS}

\subsection{Support Vector Machine (SVM) Consider the Sentences}

\begin{tabular}{|c|c|c|c|c|c|c|}
\hline S1： & THE & STUI & ENT $P$ & PASS TI & & TEST \\
\hline $\begin{array}{l}\text { Tag: } \\
\text { PUNC }\end{array}$ & DET & $\mathrm{N}$ & V & DF & & $\mathrm{N}$ \\
\hline $\begin{array}{l}\text { S2 : } \\
\text { PASS }\end{array}$ & $\begin{array}{c}\text { THE } \\
.\end{array}$ & STUI & ENTS & WAIT & FOR & THE \\
\hline $\begin{array}{l}\text { Tag : } \\
\text { PUNCT }\end{array}$ & $\begin{array}{l}\text { DET } \\
\Gamma\end{array}$ & $\mathrm{N}$ & & V & $\mathrm{P}$ & DET \\
\hline S3 : & TEAC & CHER & TEST & STUI & ENTS & . \\
\hline Tag : & $\mathrm{N}$ & & V & $\mathrm{N}$ & & PUI \\
\hline
\end{tabular}

Following is an example for the finding Input neuron [21] 


\begin{tabular}{|c|l|c|c|c|c|c|c|c|}
\hline & $\begin{array}{l}\text { T } \\
\text { H } \\
\text { E }\end{array}$ & $\begin{array}{c}\text { STUD } \\
\text { ENT }\end{array}$ & $\begin{array}{c}\text { PA } \\
\text { SS }\end{array}$ & $\begin{array}{c}\text { TE } \\
\text { ST }\end{array}$ & $\begin{array}{c}\text { W } \\
\text { AI } \\
\text { T }\end{array}$ & $\begin{array}{c}\text { F } \\
\text { O } \\
\text { R }\end{array}$ & $\begin{array}{c}\text { TEAC } \\
\text { HER }\end{array}$ & $\cdot$ \\
\hline Det & $\begin{array}{l}\frac{4}{4} \\
=1\end{array}$ & 0 & 0 & 0 & 0 & 0 & 0 & 0 \\
\hline $\mathrm{N}$ & $\frac{0}{6}$ & $\frac{3}{6}=\frac{1}{2}$ & $\frac{1}{6}$ & $\frac{1}{6}$ & $\frac{0}{6}$ & $\frac{0}{6}$ & $\frac{1}{6}$ & $\frac{0}{6}$ \\
\hline $\mathrm{V}$ & $\begin{array}{l}\frac{0}{3} \\
=0\end{array}$ & $\frac{0}{3}=0$ & $\frac{1}{3}$ & $\frac{1}{3}$ & $\frac{1}{3}$ & $\frac{0}{3}$ & $\frac{0}{3}=0$ & $\frac{0}{3}$ \\
\hline $\mathrm{P}$ & $\frac{0}{1}$ & 0 & 0 & 0 & 0 & 0 & 0 & 0 \\
\hline $\begin{array}{c}\mathrm{PU} \\
\mathrm{NC} \\
\mathrm{T}\end{array}$ & $\frac{0}{3}$ & 0 & 0 & 0 & 0 & 0 & 0 & $\frac{3}{3}$ \\
\hline$=0$ & & & & & & & $=1$ \\
\hline
\end{tabular}

Table:2 Transition probability matrix

\begin{tabular}{|c|c|c|c|c|c|c|}
\hline$\cdot$ & $\begin{array}{c}\text { Det } \\
=\mathrm{T} 1\end{array}$ & $\begin{array}{c}\mathrm{N}= \\
\mathrm{T} 2\end{array}$ & $\begin{array}{c}\mathrm{V}= \\
\mathrm{T} 3\end{array}$ & $\begin{array}{c}\mathrm{P}= \\
\mathrm{T} 4\end{array}$ & $\begin{array}{c}\text { PUNCT } \\
=\mathrm{T} 5\end{array}$ & $\mathrm{Q}_{\mathrm{F}}$ \\
\hline $\mathrm{q}_{0}$ & $2 / 3$ & $1 / 3$ & 0 & 0 & 0 & 0 \\
\hline Det & 0 & 1 & 0 & 0 & 0 & 0 \\
\hline $\mathrm{N}$ & 0 & 0 & 0 & 0 & $1 / 2$ & 0 \\
\hline $\mathrm{V}$ & $1 / 3$ & $1 / 3$ & 0 & $1 / 3$ & 0 & 0 \\
\hline $\mathrm{P}$ & 1 & 0 & 0 & 0 & 0 & 0 \\
\hline PUNCT & 0 & 0 & 0 & 0 & 0 & 1 \\
\hline
\end{tabular}

\section{Step: 1 Input neuron}

Feature vector should be produced to prepare the system. Take the primary expression of S- 1 that is, "THE" and relating Tag is "Det". The element vector can be built by choosing the primary section of table2. which covers the highlights identified with all the conceivable labels allocated to "THE". Also choosing the main segment and first line of the change framework delineated in the table 1 . The sections of the primary segment and first line of progress grid gives the probabilities of the event of the tag "Det" before the rest of the labels and after the rest of the labels separately. Joining every one of the highlights for the tag "Det" the component vector seems like $\{1,0,0,0,0,2 / 3,0,0,1 / 3,1,0,2 / 3,1 / 3,0,0,0,0\}$ So add up to 17 include neurons will be accessible in the neural systems. Also, the various element vectors for each word shows up in the preparation set. Add up to 17 words (designs) incorporating "." are accessible in the preparation set.

\section{Step: 2 Hidden neurons}

Aggregate of 17 designs are there in the database out of which number of particular neurons might be considered as Concealed neurons. The preparation set comprises of 7 unmistakable words. The 7 unmistakable words considered as focuses are $\{$ THE, STUDENT, PASS, TEST, WAIT, FOR, TEACHER, \}.

\section{Step: 3 Output neurons}

The objective of the analysis is to get a suitable tag for each word. The information database contains 5 labels viz. \{Det, N,
$\mathrm{V}, \mathrm{P}$, Punct $\}$, let us call it as $\mathrm{S}=\{1,2,3,4,5\}$. Typically, just a single yield neuron is required in the system however it won't unite. The reason is out of the extent of this book.

\section{Step: 4 Simulation process (Testing)}

Consider subjective grammatical form words to test the neural system. Self-assertive test words: \{STUDENT WAIT TEST $\}$ $=\{\mathrm{w} 1, \mathrm{w} 2, \mathrm{w} 3\}$.

There are diverse ways to deal with the issue of naming a grammatical form (POS) tag to each expression of a characteristic dialect sentence. Parts of discourse labelling is a standout amongst the most very much contemplated issues in the field of Normal Dialect Preparing (NLP).Parts of discourse labelling is the succession marking issue. Naming a POS tag to each expression of an un-clarified corpus by hand is extremely tedious which brings about finding a technique to computerize the activity. In this paper SVM Instrument is connected to the issue of grammatical form labelling for Gujarati dialect. Poslabeling can be viewed as multiclass characterization issue. This paper for the most part clarifies about how paired classifier can be utilized for multiclass characterization issue. Gujarati is composed the way it is talked. The tagset utilized as a part of this paper comprises of 10 labels. The preparation corpus comprises of 1700 words. The acquired precision is around $85 \%$ for Gujarati dialect.

\subsection{Viterbi Algorithm}

The Viterbi Calculation is the most widely recognized translating calculation utilized for Well, regardless of whether for grammatical form labeling or for discourse acknowledgment. The term Viterbi is normal in discourse and dialect handling; however, this is extremely a standard utilization of the great powerful programming calculation. The marginally rearranged adaptation of the viterbi calculation that we introduce takes as information a solitary Gee and a succession of watched words and returns the most plausible state/label grouping, together with its likelihood [19] [20].

\section{SIMULATION AND ANALYSIS}

\subsection{Training and testing data equal}

In my first investigation the preparation and testing information are same for both calculation SVM and Viterbi. In both calculations no of labels are same. We both calculations are kept running in python programming. at that point viterbi gives $100 \%$ accuracy. In SVM input neuron are 105 and concealed neuron are 325 unmistakable word and gives $59 \%$ accuracy. as a rule, SVM gives most noteworthy precision bit for my situation information base are little.

Table 1. Classification of Same datasets

\begin{tabular}{|c|c|}
\hline No of Training data $: 569$ & No of Training data $: 569$ \\
\hline No of Testing data $: 569$ & No of Testing data $: 569$ \\
\hline & Input Neuron $: 105$ \\
Hiddent Neuron $: 325$ \\
(Distinct word ).Output \\
Neuron $: 35$
\end{tabular}




\subsection{Training and Testing data are Reshuffle}

In my second investigation I influence 10 to 12 to sentences in my preparation informational index. Furthermore, again utilize viterbi and SVM. In python programming. Viterbi gives $100 \%$ exactness. What's more, in SVM I discover input neuron and shrouded neuron of 12 sentences and give $42 \%$ precision.

Table 2. Classification for Reshuffled data

\begin{tabular}{|l|l|}
\hline No of Training data:569 & No of Training data:569 \\
\hline $\begin{array}{l}\text { No of testing Data :12 } \\
\text { sentences }\end{array}$ & No of testing Data : 569 \\
\hline No of tag : 35 & $\begin{array}{l}\text { Input Neuron :10, Hidden } \\
\text { Neuron : 13, Output } \\
\text { Neuron : 35 }\end{array}$ \\
\hline Accuracy : $100 \%$ & Accuracy : 42\% \\
\hline
\end{tabular}

\subsection{Number of word available in training data set}

In my third examination are contrast for the over two experiment. in this case I make sentences however in sentences I change a few words which are not in preparing informational collection. Furthermore, check the exactness on both calculation SVM and viterbi. in viterbi utilizing python programming it gives $75 \%$ accuracy. Precision are fluctuating from sentences to sentences. be that as it may, in SVM it is hard to make grid from the sentences. For this situation I attempt in my future work.

Table 3 Classification of self generated sentences from the database

\begin{tabular}{|c|c|}
\hline No of Training data:569 & No of Training data:569 \\
\hline $\begin{array}{c}\text { No of testing Data }: 10 \\
\text { sentences }\end{array}$ & No of testing Data : 569 \\
\hline $\begin{array}{c}\text { No of tag : 35, Accuracy : } \\
70 \%\end{array}$ & - \\
\hline
\end{tabular}

\subsection{Number of words not available in training and testing data set}

In my fourth test are vary for the over three experiment. In this case I make sentences, yet all sentences are not in preparing and testing informational collection. This informational collection very surprising. In this again utilize Viterbi and SVM in python programming. Viterbi gives rough $80 \%$ accuracy yet in fluctuates from sentences to sentences. be that as it may, oh no! In svm smidgen hard to discover. so it might be tackled my next examination.

Table 4 Classification of self generated sentences with some external words

\begin{tabular}{|c|c|}
\hline No of Training data:569 & No of Training data:569 \\
\hline No of testing Data : 569 & No of testing Data : 569 \\
\hline $\begin{array}{c}\text { No of tag : 35, Accuracy : } \\
80 \%\end{array}$ & \\
\hline
\end{tabular}

\subsection{Graphical Representation of Error}

For table 1, in graphical portrayal I make a table in claim references and check specific all tag for SVM in how much time wrong. For illustration NN (thing) aren't right 10 times, likewise PUNC (punctuation) aren't right 56 times etc.... also, draw the chart. What's more, in viterbi there is no blunder. so its charts are straight.

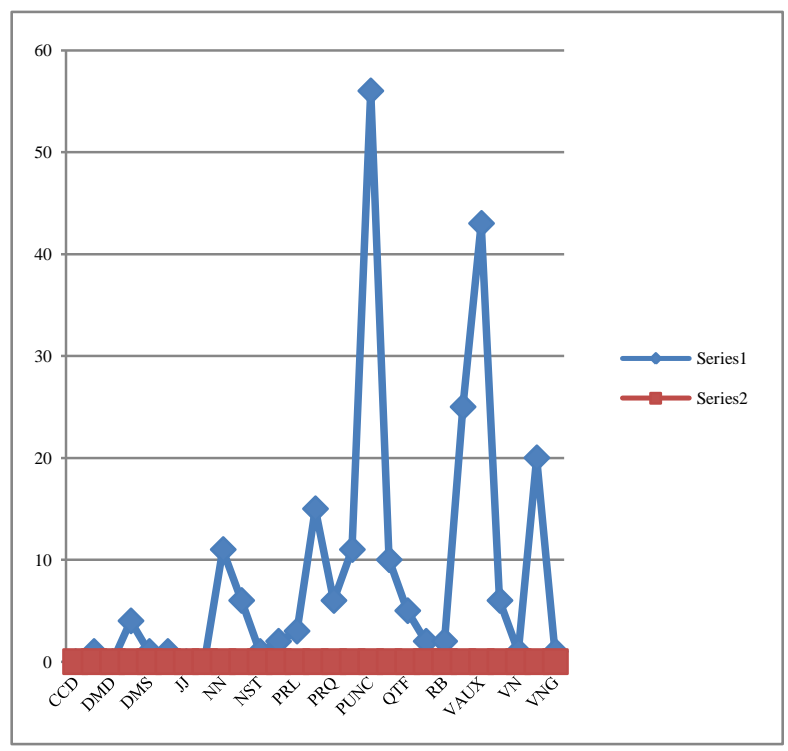

Fig 2 Tagwise Error Analysis

\section{CONCLUSION}

Investigations delineated in four tables in segment, viterbi performs reliably better in all the four classes when contrasted with SVM. As highlighted in figure 2, In 56 cases SVM does not label accentuation stamps legitimately. In the greater part of the cases it is misclassified with VM TAG. Anyway, that isn't the situation for Viterbi.

\section{REFERENCES}

[1]. Aं M. T. M. and B D. C. V., "Survey : Natural Language Parsing For Indian Languages," 2015.

[2]. R. Abstract, P. O. Speech, N. L. Processing, E. Schedule, I. Constitution, and I. Constitution, "Parts Of Speech Tagger for Maithili Language Using HMM," vol. 7, no. 4, pp. 206-211, 2018.

[3]. P. J. Antony and K. P. Soman, "Ke[1] P. J. Antony and K. P. Soman, 'Kernel based part of speech tagger for Kannada,' 2010 Int. Conf. Mach. Learn. Cybern. ICMLC 2010, vol. 4, no. July, pp. 2139-2144, 2010.rnel based part of speech tagger for Kannada," 2010 Int. Conf. Mach. Learn. Cybern. ICMLC 2010, vol. 4, no. July, pp. 2139-2144, 2010.

[4]. A. Bharati, M. Gupta, V. Yadav, K. Gali, and D. M. Sharma, "Simple parser for Indian languages in a dependency framework," Proc. Third Linguist. Annot. Work., no. August, pp. 162-165, 2009.

[5]. B. R. Das, S. Sahoo, C. S. Panda, and S. Patnaik, "Part of speech tagging in odia using support vector machine," Procedia Comput. Sci., vol. 48, no. C, pp. 507-512, 2015.

[6]. P. S. Dholakia and M. M. Yoonus, "Rule Based Approach for the Transition of Tagsets to Build the $\{$ POS $\}$ Annotated Corpus," Int. $\{\mathrm{J}\}$ ournal $\{\mathrm{A}\}$ dvanced 
$\{\mathrm{R}\}$ esearch $\{\mathrm{C}\}$ omputer $\quad\{\mathrm{C}\}$ ommunication \{E\}ngineering, vol. 3, no. 7, pp. 7417-7422, 2014.

[7]. A. Ekbal and S. Bandyopadhyay, "Part of speech tagging in Bengali using Support vector Machine," Proc. - 11th Int. Conf. Inf. Technol. ICIT 2008, pp. 106-111, 2008.

[8]. S. Haykin, Neural Networks and Learning Machines, vol. 3. 2008.

[9]. S. Journal and I. Factor, "International Journal of Advance Engineering and Research Development," pp. 464-467, 2015.

[10].M. T. Makwana and D. C. Vegda, "Survey:Natural Language Parsing For Indian Languages."

[11].G. Mcdonald and C. Macdonald, "A Study of SVM Kernel Functions for Sensitivity Classification Ensembles with POS Sequences," no. June, pp. 7-11, 2017.

[12].A. Mukherjee, S. Kübler, and M. Scheutz, "POS Tagging Experts via Topic Modeling," Proc. 13th Int. Conf. Nat. Lang. Process., pp. 120-128, 2016.

[13].A. Nietzio, "Support Vector Machines for Part-of-Speech Tagging," pp. 5-8, 2002.

[14].K. Nongmeikapam, "Manipuri Chunking: An Incremental Model with POS and RMWE," no.
December, pp. 277-286, 2014.

[15].K. Nongmeikapam and S. Bandyopadhyay, "Genetic Algorithm (GA) Implementation for Feature Selection in Manipuri POS Tagging," Proc. 13th Int. Conf. Nat. Lang. Process., no. December, pp. 267-274, 2016.

[16].C. Patel and K. Gali, "Part-Of-Speech Tagging for $\{\mathrm{G}\}$ ujarati Using Conditional Random Fields," Proc. IJCNLP-08 Work. NLP Less Privil. Lang., no. January, pp. 117-122, 2008.

[17].B. Plank, "Natural Language Processing : Introduction to Syntactic Parsing," 2012.

[18].T. B. Shahi, "Support Vector Machines based Part of Speech Tagging for Nepali Text," vol. 70, no. 24, pp. 38-42, 2013.

[19].Manisha prajapati Yajnik Archit, "Part of Speech Tagging Using Statistical Approach for Gujrati Text," vol. 11, no. 1, pp. 76-79, 2017.

[20].A. Yajnik, "Part of Speech Tagging Using Statistical Approach for Nepali Text," vol. 11, no. 1, pp. 76-79, 2017.

[21].Shigeo Abe ,Yasuyuki Tajiri, Ryosuke Yabuwaki and Takuya Kitamura, "Feature extrction using SVM." ICON NIP, pp. 1-8, 2010. 